\section{What is IUGS?}

The International Union of Geological Sciences (IUGS) is one of the largest and most active non-governmental scientific organizations in the world. Founded in 1961, IUGS is a member of the International Council for Science (ICSU). IUGS promotes and encourages the study of geological problems, especially those of worldwide significance, and supports and facilitates international and interdisciplinary cooperation in the earth sciences. to:

At present IUGS gives special consideration

- Initiatives related to the identification and assessment of energy and mineral resources;

- Global change;

- Geologic hazards;

- Environmental geology;

- Geological education and capacity building, and

- Geoinformation.

IUGS Commissions, Committees, Task Groups and Boards are concerned with a wide range of geologic research of direct interest to governments, industry, and academic groups within the earth sciences. IUGS believes that it is of mutual benefit to establish close links with other organizations engaged in geoscience activities, and especially those organizations whose work relates to some of the major activities of IUGS.

\section{How did IUGS originate?}

The Union was founded in March of 1961 in response to a need to coordinate geoscientific international research programs on a continuing basis. Geoscientists felt that a mechanism was required to take action on global geological problems between the International Geological Congresses, traditionally held every four years.

IUGS was also to serve as a vital link in solving problems requiring interdisciplinary input from other international scientific unions operating under the aegis of the International Council of Scientific Unions. Compared to these, IUGS was a 'latecomer,' although some of its responsibilities had been carried out by the International Geological Congress-a venerable institution over a century old.

Since 1961, IUGS has experienced rapid growth in membership, scientific scope and expertise, as well as international prestige.

\section{IUGS beyond 1961-2012}

IUGS has been actively developing new goals and directions for the next 50 years under its evolving strategic plan. The Union continues to work in a collaborative fashion with other Earth science unions and our partner organizations worldwide to achieve a balanced program. Additional high priorities are in the areas of Earth science education and technology transfer between the developed and developing countries.

\section{What does IUGS do?}

IUGS fosters dialogue and communication among the various specialists in Earth sciences around the world. It achieves this by organizing international projects and meetings, sponsoring symposia and scientific field trips, and producing publications.

Topics addressed span the gamut from fundamental research to its economic and industrial applications, from scientific, environmental and social issues to educational and developmental problems. For example, IUGS is currently involved in:

- identifying and defining the problems critical to an improved understanding of terrestrial and planetary geological processes;

- encouraging formulation and testing of new geological concepts, models, and methodologies;

- focusing effort internationally on the study of critical economic or environmental problems whose resolution may depend on an understanding of geology;

- fostering international agreement on nomenclature and classification in several geoscientific disciplines such as stratigraphy, petrology and tectonics;

- strengthening mechanisms for facilitating international cooperation in geological research and exchange;

- improving publication, dissemination, and use of geological information internationally;

- encouraging new relationships between and among disciplines of science that relate to geology worldwide;

- attracting competent students and research workers who will devote their attention to geology, and to stimulate excellent education for students interested in geology;

- fostering an increased awareness among individual scientists worldwide of what programs are being carried out in geology in each country;

- promoting public understanding and appreciation of the planet Earth, its environment in space, and their study; and

- furthering the public welfare by assuring that geology makes appropriate contribution to public policy decisions of an international nature.

\section{IUGS Executive Committee}

\section{IUGS President}

Professor Alberto C. RICCARDI

Facultad de Ciencias Naturales y Museo

Universidad Nacional de la Plata

Paseo del Bosque s/n

1900 La Plata, Buenos Aires,

Argentina

T: +54 221425 7744/9161/9638 (Ext.114)

F: +54 2114257527

E: riccardi@fcnym.unlp.edu.ar

\section{IUGS Secretary General}

Dr. Peter BOBROWSKY

Geological Survey of Canada

601 Booth Street

Ottawa Ontario K1A 0E8,

Canada

T: +1 6139470333

F: +16139920190

E: pbobrows@nrcan.gc.ca
IUGS Treasurer

Prof. William CAVAZZA

Dept. of Earth and Geoenvironmental Sciences

University of Bologna

Piazza di Porta San Donato 1

IT-40126 Bologna, Italy

T: +390512094939

F: +390512094904

E:william.cavazza@unibo.it

\section{IUGS Past President}

Prof. ZHANG Hongren

64 Funei Dajie

100812 Beijing, China

$\mathrm{T}:+861066557485$

F: +861066557473

E: zhr@yahoo.com

\section{IUGS Vice President}

Dr. Jacques CHARVET

Orléans University

ISTO, Earth Sciences

B.P. 6759 , FR 45067

Orléans Cedex 2, France

T: +33147072284

F: +33143369518

E: jacques.charvet@univ-orleans.fr

\section{IUGS Vice President}

Prof. GEREL Ochir

Department of Geology and Mineralogy

Mongolian University of Science and Technology,

P.O. 46, Box 520

Ulaanbaatar 210646, Mongolia

T: +976 $11324500,+97611326425$

F: +976 11312 291, +976113241218

E: gerel@must.edu.mn; gerel_17@yahoo.com

IUGS Councillor(2008-2012)

Dr. Colin SIMPSON

73 Beasley Street

Torrens ACT 2607, Australia

$\mathrm{T}:+61262863548$

F: +61262867548

E: simpsons@grapevine.com.au

IUGS Councillor (2008-2012)

Professor Ezzoura ERRAMI

Département de Géologie

Faculté des Sciences

Université Chouaïb Doukkali, BP. 20

24000 El Jadida, Morocco

T: +212 68038116

F: +212 23342187

E: erramiezzoura@yahoo.fr

IUGS Councillor (2010-2014)

Ms. Wesley HILL

The Geological Society of America

P.O. Box 9140

Boulder, CO 80301, USA

$\mathrm{T}:+13033571005$

F: +1 3033571070

E:whill@geosociety.org

IUGS Councillor (2010-2014)

Prof. Sampat Kumar TANDON

University of Dehli, Department of Geology

A-2; 29 -31 Chhatra Marg

Dehli - 110007, India

T: +91 (0) 1127667899

E: sktand@rediffmail.com 
IUGS Secretariat

http://www.iugs.org

U.S. Geological Survey

MS-917, National Center

12201 Sunrise Valley Drive

Reston, VA 20192

T: +01-703-648-6050

F: +01-703-648-4227

E: iugs@usgs.gov

\section{IUGS COMMITTEES}

\section{Nominating Committee}

\section{Chairman}

Prof. ZHANG Hongren

64 Funei Dajie, 100812 Beijing, China

T: +861066557485

F: +861066557473

E:zhr@yahoo.com

\section{Members}

Dr. Marita BRADSHAW (Australia)

E: marita.bradshaw@ga.gov.au

Dr. Elena CENTENO-GARCIA (Mexico)

E: centeno@servidor.unam.mx

Dr. Peadar MCARDLE (Ireland)

E: Peadar.Mcardle@gsi.ie

Dr. Jonas SATKUNAS (Lithuania)

E: jonas.satkunas@lgt.lt

Prof. Ryo MATSUMOTO (Japan)

E:ryo@eps.s.u-tokyo.ac.jp

\section{Publication Committee}

\section{Chairman}

Dr. Fred SPILHAUS

PO Box 53024, Washington DC 20036, USA

T: +01-301-299-8781

E: Fspilhaus@alum.mit.edu

\section{Members}

Prof. Susana DAMBORENEA (Argentina)

E: sdambore@fcnym.unlp.edu.ar

Dr. Mudlappa JAYANANDA (India)

E: episodes.editor@gmail.com;

Dr. Michael THOMSON

E:m.thomson@stone-house.demon.co.uk

Dr. Zhenyu YANG (China)

E: paleo_mag@yahoo.com.cn

\section{International Geological Congress}

\author{
$34^{\text {th }}$ INTERNATIONAL GEOLOGICAL \\ CONGRESS \\ Brisbane, Australia- August 2-10, 2012 \\ http://www.34igc.org \\ President \\ Dr. Neil WILLIAMS \\ E: williamsgeoscience@grapevine.com.au
}

Secretary General

Dr. Ian LAMBERT

Geoscience Australia

GPO Box 378

Canberra ACT 2601, Australia

T: +61 262499965

F: +61262499965

E: ian.lambert@ga.gov.au

$35^{\text {th }}$ INTERNATIONAL GEOLOGICAL CONGRESS

Capetown, S. Africa - August 6-14, 2016

http://www.35igc.org

Dr. Danie BARNARDO

E: barnardo@geoscience.org.za

\section{International Council of Science (ICSU)}

http://www.icsu.org

\section{President}

Prof. Catherine BRECHIGNAC

CNRS, Campus Gérard-Mégie

3 rue Michel-Ange

F-75794 Paris cedex 16, France

T: +33144964000

F: +33144965390

E: catherine.brechignac@cnrs-dir.fr

\section{Executive Director}

Dr. Steven WILSON

International Council of Science

5 rue Auguste Vacquerie

F-75016 Paris, France

$\mathrm{T}:+33(00) 145250329$

direct $+33(00) 145250701$

F: +33 (00) 142889431

E: secretariat@icsu.org

\section{Episodes}

Journal of International Geoscience http://www.episodes.org

\section{Editor}

Prof. M. JAYANANDA

Department of Geology

Centre of Advanced Studies

University of Delhi,

Delhi-110007

India

T. $+91-9560714425$

F: +91- 9448865700

E:episodes.editor@gmail.com

E:mjayananda@rediffmail.com

\section{Associate Editors}

Dr. Norm CATTO (Canada)

E: ncatto@mun.ca

Prof. Sierd CLOETINGH (Netherlands)

E: cloeting@geo.vu.nl

Prof. Umberto CORDANI (Brazil)

E: ucordani@usp.br

Dr. I. W. DALZIEL (USA)

E: ian@ig.utexas.edu
Prof. Robert N. GINSBURG (USA)

E:rginsburg@rsmas.miami.edu

Dr. Alexander O. GLIKO (Russia)

E: gliko@uipe-ras.scgis.ru

Prof. Felix M. GRADSTEIN (Norway)

E: felix.gradstein@nhm.uio.no

Prof. Richard A. GRIEVE (Canada)

E: rgrieve@nrcan.gc.ca

Prof. Bor-ming JAHN (Taipei, China)

E: jahn@earth.sinica.edu.tw

Dr. KINZELBACH (Switzerland)

E: kinzelbach@ihw.baug.ethz.ch

Dr. David R. OLDROYD (Australia)

E:doldroyd@optushome.com.au

Prof. Jane PLANT (UK)

E: jane.plant@imperial.ac.uk

Prof. Isabella PREMOLI-SILVA (Italy)

E: Isabella.Premoli@unimi.it

Dr. Kalachand SAIN (India)

E: kalachandsain@yahoo.com

Prof. S. UYEDA (Japan)

E: suyeda@riken.gp.jp

Dr. Maarten de WIT (South Africa)

E:maarten@cigoes.ac.za

Dr. HONG-FU Yin (China)

E: hfyin@dns.cug.edu.cn

\section{IUGS COMMISSIONS}

COMMISSION ON THE MANAGEMENT \& APPLICATION OF GEOSCIENCE INFORMATION (CGI)

http://www.cgi-iugs.org

\section{Chairman}

Dr. Kristine ASCH

BGR, Stilleweg 2

D-30655 Hannover, Germany

T: +49 5116433324

F: +495116433782

E: Kristine.Asch@bgr.de

Secretary General

Mr. Ian JACKSON

British Geological Survey

Kinsley Durham Centre

Keyworth, Nottingham NG12 5GG

United Kingdom

T: +44 1159363214

F: +44 1159363269

E: ij@bgs.ac.uk

\section{Treasurer}

Dr. François ROBIDA

3, Av. Claude Guillemin

BRGM

BP 36009, F-45060 Orléans Cedex

France 
T: +33238643132

F: +33238 643652

E: f.robida@brgm.fr

Members

Dr. Gabriel ASATO

GIS Unit - SEGEMAR

E: g_asato2000@yahoo.com

Dr. Peter BAUMANN (Germany)

Jacobs University

E : p.baumann@jacobs-university.de

Dr. Henry John BROOME (Canada)

Natural Resources Canada

E: broome@nrcan.gc.ca

Dr. Anna-Karren NGUNO (Namibia)

E: anguno@mme.gov.na

Dr. David PERCY (USA)

Portland State University

E: percyd@pdx.edu

Dr. Bruce SIMONS (Australia)

E: bruce.simons@dpi.vic.gov.au

Dr. Koji WAKITA (Japan)

E: koji-wakita@aist.go.jp

Dr. WANG Jun (China)

E:wangj29@126.com

Member Elect

Linda GUNDERSEN (USA)

Lgundersen@usgs.gov

GEOSCIENCE FOR ENVIRONMENTAL MANAGEMENT (GEM)

http:// www.iugs-gem.org

Chair

Dr. Kevin TELMER, Associate Professor

School of Earth and Ocean Sciences

University of Victoria

PO Box 3065 STN CSC

Victoria, BC, Canada V8W 3V6

Tel: +1 250-472-4182

Main Office +1 250 721-6120

Fax: +1 250-721-6200

ktelmer@uvic.ca

www.seos.uvic.ca

\section{Vice Chair}

Dr. Qingcheng HE

Deputy Chief Geologist

China Institute of Geo-Environmental

Monitoring, China Geological Survey (CGS)

No.20 Dahuisi, Haidian District

Beijing 100081, China

Tel: +86-10-62171221

Fax: +86-10-62173426

E: heqc@mail.cigem.gov.cn

E: heqc@public.bta.net.cn

www.cigem.gov.cn

\section{Secretary General}

Dr. Brian MARKER

40 Kingsdown Avenue,

London, W13 9PT, UK

Tel: + 2085675946

E: brian@amarker.freeserve.co.uk
Treasurer

Dr. Kaj LAX

Geological Survey of Sweden

Div. of Environment and Geochemistry

P.O. Box 670, SE-751 28 Uppsala, Sweden

Tel: +46 (0)18 179394

Mob.: +46 (0)702246385

Fax: +46 (0)18179210

E:kaj.lax@sgu.se,kaj.lax@gmail.com

\section{Information Coordinator}

Daniel STAPPER (Canada)

E: daniel@artisinalgold.org

\section{Officers}

Officer for Africa

Dr. Ben MAPANI (Namibia)

E: bmapani@unam.na

www.unam.na

\section{Officer for Western Africa}

Dr. Sulemana Al-HASSAN (Ghana)

E: shassangh@yahoo.com

Officer for the Caribbean and South America Dr. Jose A. CENTENO (USA)

E: jacenteno@comcast.net

E: tonycent@comcast.net

Officers for Europe

Prof. Eduardo da SILVA (Portugal)

E: eafsilva@ua.pt

http://www.ua.pt/

Dr. Laurance DONNELLY (UK)

E: ldonnelly@wardell-armstrong.com

Website: www.wardell-armstrong.com

Prof. Marek GRANICZY (Poland)

E: marek.graniczny@pgi.gov.pl

E: http://www.pgi.gov.pl/pgi_en/

\section{Officer for Europe \& Scandanavia}

Dr. Philipp SCHMIDT-THOMÉ (Finland)

E: philipp.schmidt-thome@gtk.fi

http://en.gtk.fil

\section{Officer for Eastern Europe \& Eurasia}

Prof. Igor ZEKSTER (Russia)

E: zektser@aqua.laser.ru,

Website: http://www.ras.ru/

\section{Officer for South America}

Prof. Bernardino de FIGUEIREDO (Brazil)

E: berna@ige.unicamp.br

Website: http://www.ige.unicamp.br/

Officer for North America

Dr. David LIVERMAN (Canada)

E: dliverman@gov.nl.ca

http://www.nr.gov.nl.ca/mines\&en/geosurvey/

Dr. Monica JARAMILLO (Canada)

E: monica.jaramillo@bchydro.com

Officer for Asia

Dr. Mihir DEB (India)

E: mihirdeb@gmail.com

Officers of Japan Branch

Mr. Kunio FURUNO (Japan)

E: k.hrn50@ma.pref.chiba.lg.jp
Professor Hisashi NIREI (Japan)

E: nireihisashi@msn.com

INTERNATIONAL COMMISSION ON STRATIGRAPHY (ICS)

http://www.stratigraphy.org

Chairman

Dr. Stanley FINNEY

Dept. of Geological Sciences

California State University at Long Beach

1250 Bellflower Blvd

Long Beach, CA 90840, USA

$\mathrm{T}:+15629858637$

F: +1 5629858638

E: scfinney@csulb.edu

Secretary General

Professor Paul R. BOWN

Department of Earth Sciences

University College London

Gower Street, London WC1E 6BT

United Kingdom

T: +44020 76792431

F: +4402076792685

E: p.bown@ucl.ac.uk

Vice Chair

Dr. Shanchi PENG

Nanjing Institute of Geology and

Palaeontology

39 East Beijing Road

210008 Nanjing, China

T: +86 (0)25 3282169

F: +86 (0)25 3357026

E: scpeng@nigpas.ac.cn

\section{STRATIGRAPHY SUBCOMMISSIONS}

Subcommission on Precambrian Stratigraphy http://www.stratigraphy.org/bak/precambrian

\section{Chair}

Dr. Martin VAN KRANENDONK

Geological Survey of Western Australia

Mineral House, 100 Plain Street

East Perth, Western Australia 6004

Australia

T: +61 $89222-3631$

F: +61 $89222-3633$

E: martin.vankranendonk@doir.wa.gov.au

Subcommission on Neoprotozoic Stratigraphy (Ediacarn-Cyrogenian)

http://www.stratigraphy.org/bak/ediacaran/

Chair

Dr. James GEHLING

South Australian Museum

North Terrace

Adelaide, 5000 South Australia

T: +61 882077441

E:wjgehling@ozemail.com

Subcommission on Cambrian Stratigraphy http://www.palaeontologie.uni-wuerzburg.del casul-R.htm

Chair

Dr. Shanchi PENG

Nanjing Institute of Geology and Palaeontology 39 East Beijing Road 
210008 Nanjing, China

$\mathrm{T}:+86(0) 253282169$

F: $+86(0) 253357026$

E: scpeng@nigpas.ac.cn

Subcommission on Ordovician Stratigraphy

http://www.ordovician.cn/index.asp

\section{Chair}

Prof. David A.T. HARPER

Geological Museum

Collection of Invertebrate Palaeontology

Øster Voldgade 5-7

DK-1350 Copenhagen K,

Denmark

$\mathrm{T}:+4535322371$

F: +4535322325

E: DHarper@snm.ku.dk

Subcommission on Silurian Stratigraphy http://www.silurian.cn/home.asp

\section{Chair}

Dr. Michael MELCHIN

St. Francis Xavier University

Department of Earth Science

P.O. Box 5000

Antigonish, Nova Scotia B2G 2W5

Canada

T: +1 9028675177

F: +1 9028672414

E: mmelchin@stfx.ca

Subcommission on Devonian Stratigraphy http://www.unica.it/sds/

\section{Chair}

Dr. Thomas BECKER

Geologisch-Paläeontogisches Institut

Westfalische Wilhelm-Universität

Correnstrasse 24, D-48149 Münster

Germany

T: $+4925183-3395$

F: +49 251 83-33968

E: rbecker@uni-muenster.de

Subcommission on Carboniferous Stratigraphy http://www.nigpas.ac.cn/carboniferous/web/ index.asp

\section{Chair}

Professor Barry RICHARDS

Geological Survey of Canada-Calgary

3303-33 St. NW

Calgary, Alberta T3Z 2S6

Canada

E: brichard@nrcan.gc.ca

Subcommission on Permian Stratigraphy http://www.nigpas.ac.cn/permian/web/ index.asp

\section{Chair}

Dr. Charles HENDERSON

University of Calgary

Department of Geoscience

2500 University Drive NW

Calgary, Alberta T2N 1N4,

Canada

$\mathrm{T}:+14032206170$

F: +1 4032200074

E: charles.henderson@ucalgary.ca
Subcommission on Triassic Stratigraphy

http://paleo.cortland.edu/sts/

\section{Chair}

Dr. Marco BALINI

Dipartimento di Scienze della Terra

Università degli Studi di Milano

via Mangiagalli 34

I-20133 Milano, Italy

E: marco.balini@unimi.it

Subcommission on Jurassic Stratigraphy http://jurassic.earth.ox.ac.uk/

\section{Chair}

Dr Jozsef PALFY

Hungarian Natural History Museum

Department of Geology and Paleontology

PO Box 137

1431 Budapest, Hungary

T: +3613383905

F: +3613382728

E: palfy@nhmus.hu

$\underline{\text { Subcommission on Cretaceous Stratigraphy }}$

\section{Chair}

Professor Isabella Premoli SILVA

Dip. Scienze della Terra, "Ardito Desio"

Universita degli Studi di Milano

Via Mangiagalli 34

I-20133 Milan, Italy

T: +3902 50315528

F: +390250315494

E: Isabella.Premoli@unimi.it

\section{Subcommission on Paleogene Stratigraphy}

http://wzar.unizar.es/isps/

\section{Chair}

Dr. Eustoquio MOLINA

Departamento de Ciencias de la Tierra

Universidad de Zaragoza

Calle Pedro Cerbuna 12,

E-50009 Zaragoza, Spain

$\mathrm{T}:+34976761077$

F: +34 976761106

E: emolina@unizar.es

\section{Subcommission on Neogene Stratigraphy} http://www.geo.uu.nl/sns/

\section{Chair}

Prof. Frederik HILGEN

University of Utrecht

Faculty of Earth Sciences

Budapestlaan 4, POB 80021

3508 TA Utrecht, The Netherlands

T: +31302535186

F: +31302532648

E: fhilgen@geo.uu.nl

Subcommission on Quaternary Stratigraphy http://www.quaternary.stratigraphy.org.uk/

\section{Chair}

Dr. Philip GIBBARD

Godwin Institute of Quaternary Research

Department of Geography

Downing Street, University of Cambridge

Cambridge CB2 3EN, United Kingdom

E:plg1@cam.ac.uk
Subcommission on Stratigraphic Classification http:// users.unimi.it/issc

Chair

Dr. Brian PRATT

University of Saskathewan

Dept. of Geological Sciences

114 Science Place, Saskatoon

Saskatchewan S7N 5E2, Canada

$\mathrm{T}:+13069665725$

F: +13069668593

E: brian.pratt@usask.ca

INTERNATIONAL COMMISSION ON THE HISTORY OF GEOLOGICAL SCIENCES (INHIGEO)

http://www.inhigeo.org

\section{President}

Dr. Silvia FIGUEIRÔA

Institute of Geosciences

University of Campinas (UNICAMP)

P.O. Box 6152

13083-970 Campinas-SP, Brazil

$\mathrm{T}:+55$ (19) 35214553

F: +55 (19) 35211562

E: figueiroa@ige.unicamp.br

Secretary General

Dr. Barry COOPER

Barbara Hardy Institute

School of Natural and Built Environments

University of South Australia (Mawson Lakes

Campus)

GPO Box 2471

Adelaide SA 5001, Australia

E: barry.cooper@unisa.edu.au

Vice President, Asia

Prof. ZHANG Jiuchen

Institute for the History of Natural Science

Chinese Academy of Science

137, Chao Nei Avenue

100010 Beijing, China

$\mathrm{T}:+86108402-7627$

E:jhbz@ihns.ac.cn; zhangjiuchen9@sina.com

Vice President, Australia

Prof. David OLDROYD

University of New South Wales

28 Cassandra Avenue

St. Ives, NEW SOUTH WALES 2075

Australia

E: doldroyd@optushome.com.au

Vice President, Europe

Dr. Martina KOELBL-EBERT

Jura-Museum

Burgstrasse 19

Willibaldsburg

D 85072 Eichstätt, Germany

T: +49 84 218-9609

E: koelbl-ebert@jura-museum.de

Vice President, Latin America

Mr. Gerardo SOTO

Instituto Costarricense de Electricidad

Apartado 360-2350

San Francisco de dos Ríos

1000 San Jose, Costa Rica

T: +50 6 219-4051

E: katomirodriguez@yahoo.com 
Vice President, North America

Dr. Gregory GOOD

American Institute of Physics

One Physics Ellipse

College Park, MD 20740-3843 USA

T: +1 3012093174

E:ggood@aip.org

Past President

Prof. Philippe TAQUET (France)

Email:philippe.taquet@wanadoo.fr

IUGS COMMISSION ON EDUCATION, TRAINING AND TECH TRANSFER (COGE)

http://www.geoed.com.au/IUGSeducation/ indexcomm.html

\section{Chairman}

Dr. Jesus Martinez FRIAS

Centro de Astrobiologia (CSIC-INTA)

Madrid, Spain

E: jmfrias@cab.inta-csic.es

Vice Chairman

Professor Chris KING

Keele University

United Kingdom

c.j.h.king@educ.keele.ac.uk

Secretary General

Ms. Jennifer NOCERINO

The Geological Society of America

3300 Penrose Place

Boulder, CO 80301 USA

Phone: 303-357-1036

$\mathrm{T}:+13033571036$

F: +1 3033571070

E: jnocerino@geosociety.org

COMMISSION ON TECTONICS AND STRUCTURAL GEOLOGY (TECTASK)

http://www.tectask.org

\section{Chair}

Professor Dr. Cees W. PASSCHIER

Institut für Geowissenschaften

Johannes Gutenberg University

Becherweg 21

DE 55099 Mainz, Germany

T: +4961313923217

F: +4961313923863

E: cpasschi@mail.uni-mainz.de

\section{Vice Chairman}

Dr. Mark JESSELL, IRD LMTG

Universite Paul-Sabatier

14 avenue Edouard Belin

Toulouse 31400 CEDEX,

France

T: +33(0)5 61332647

F: +33 561332560

E: mark.jessell@lmtg.obs-mip.fr

Website: www.lmtg.obs-mip.fr/user/mjessell

\section{Secretary General}

Dr. Hermann LEBIT

Houston, TX 77056-2725,

USA

T: +17132962698

F: +1 7132964310

E: lebit@sbcglobal.net

\section{TASK GROUPS}

GLOBAL GEOCHEMICAL BASELINES
(TGGGB)
http://www.globalgeochemicalbaselines.eu/

Leader

Dr. David B. SMITH, USGS

Mailstop 973 - Denver Federal Center

Denver, Colorado 80225, USA

T: +1 3032361849

F: +1 3032363200

E:dsmith@usgs.gov

Leader

Dr. Xueqiu WANG

Institute of Geophysical and Geochemical

Exploration, 84 Golden Road

Langfang, Hebei 065000, China

$\mathrm{T}:+86-316-226-7629$

F: +86-316-221-2744

E: wangxueqiu@igge.cn

Scientific Secretary

Mr. Shaun REEDER

British Geological Survey, Keyworth

Nottingham NG12 5GG, UK

F: +44 (0) 1159363261

$\mathrm{T}:+44$ (0) 1159363523

E: shaun.reeder@hotmail.co.uk

\section{TASK GROUP GLOBAL GEOSCIENCE WORKFORCE (TGGGW)}

Secretary General

Dr. Christopher KEANE

American Geosciences Institute

4220 King Street

Alexandria, VA 22302, USA

$\mathrm{T}:+1$ 703-379-2480

F: +1 703-379-7563

E: Keane@agiweb.org

TASK GROUP ON GEOHERITAGE (TGG)

\section{Chairman}

Professor Patrick DE WEVER

Geology Department, National Museum of

Natural History, 43 rue Buffon F-75 005

Paris Cedex 05, France

Tel: +330140793482

Email: pdewever@mnhn.fr

\section{HERITAGE STONE TASK GROUP (HSTG)}

President (also ex officio as Chairman of IAEG C-10)

Dr. Bjorn SCHOUENBORG

Materials Department, SP Swedish National

Testing and Research , Institute, Box 857,

SE-501, 15 Boras, Sweden

E: bjorn.schouenborg@cbi.se

\section{Secretary General}

Dr. Barry J. COOPER

Barbara Hardy Institute

School of Natural and Built Environments

University of South Australia (Mawson Lakes

Campus), GPO Box 2471

Adelaide SA 5001, AUSTRALIA

E: barry.cooper@unisa.edu.au
Vice President of Southern Europe

Professor Dolores PEREIRA

E:mdp@usal.es

Vice President of Central Europe

Dr. Sabrina KRAMAR

E: Sabina.kramar@rescen.si

Vice President Western Europe

Professor Dr. Jan ELSEN

E: jan.elsen@geo.kuleuven.be

Vice President North America

Dr. Joseph T. HANNIBAL

E: jhanniba@cmnh.org

Vice President North America

Professor Brian R. PRATT

E: brian.pratt@usask.ca

Vice President North America

Dr. Nelson R. SHAFFER

E: shaffern@indiana.edu

Vice President South America

Prof. Fabiano Cabañas NAVARRO

E: fabiano.navarro@unifal-mg.edu.br

Vice President East Asia

Dr. Hirokazu KATO

E: h.katou@aist.go.jp

Vice President South Asia

Dr. Harel THOMAS

E: harelthomas@yahoo.com

Vice President Africa

Dr. Phil PAIGE-GREENE

E: ppaigegr@csir.co.za

Member

Dr. Brian R. MARKER

E:brian@amarker.freeserve.co.uk

IUGS/IUPAC TASK GROUP ON ISOTOPES AND GEOCHRONOLOGY (TGIG)

Chair

Professor Igor VILLA

Institute for Geology

Baltzerstrasse 3 ,

CH 3012 Bern

Switzerland

$\mathrm{T}:+41-31-631-8777$

F: +41-31-631-4843

E: igor@geo.unibe.ch

Secretary General

Professor Paul R. RENNE

Berkeley Geochronology Center

2455 Ridge Road,

Berkeley, CA 94720

USA

$\mathrm{T}:+15106449200$

E: prenne@bgc.org

IUPAC Members

Prof. Mauro BONARDI

Dr. Paul DEBIEVE

Dr. Ales FAJGELJ

Dr. Norman HOLDEN

Dr. Dunyi LIU 


\section{JOINT PROGRAMMES \\ IUGS/UNESCO INTERNATIONAL GEOSCIENCE PROGRAMME (IGCP) http://unesco.org/science/earth/igcp/ index_igcp.html \\ IGCP Secretariat \\ Dr. Margarete PATZAK \\ Division of Ecological and Earth Sciences \\ UNESCO, 1, Rue Miollis \\ 75732 Paris, Cedex 15, France \\ T: +33145684118 \\ F: +33145685822 \\ E: igcp@unesco.org}

\section{IUGS/UNESCO GEOLOGICAL APPLICATIONS OF REMOTE SENSING (GARS)}

\section{Chairman}

Professor Stuart MARSH

Head, Geoscience Technologies

British Geological Survey

Keyworth, Nottingham NG12 5GG

United Kingdom

T: +44 (0)115936 3452

F: +44 (0)115936 3200

E: shm@bgs.ac.uk

\section{Secretary}

Ms. Sarah GAINES

Division of Ecological \& Earth Sciences,

UNESCO

7 Place de Fontenoy,

F-75700 Paris, France

T: +33145684117

F: +33145685822

E: s.gaines@unesco.org

\section{INTERNATIONAL LITHOSPHERE \\ PROGRAM (ILP)}

http://www.sclilp.org/

\section{President}

Prof. Sierd CLOETINGH

Department of Earth Sciences,

Faculty of Geosciences, Utrecht University

3584CD Utrecht, The Netherlands

T: +31 204447341

F: +31 206462457

E: sierd.cloetingh@uu.nl

\section{Secretary General}

Professor Dr. Roland OBERHÄNSLI

Institute of Earth \& Environmental Sciences

University Potsdam

Karl Liebknecht Strasse 24

D-14476 Potsdam, Germany

T: +49 3319775808

F: +49 3319775700

E: secretariat-ILP@gfz-potsdam.de

\section{FORENSIC GEOLOGY INITIATIVE (FGI)}

\section{Chairman}

Dr. Laurance DONNELLY

Wardell Armstrong LLP

2 The Avenue, Leigh

Greater Manchester,

United Kingdom
T: +01942 260101

F: +4773921620

M: +07970038 236

E: ldonnelly@wardell-armstrong.com

\section{AFFILIATED ORGANIZATIONS}

\section{ASSOCIATION OF APPLIED GEOCHEMISTS (AAG)}

\section{President}

Paul MORRIS, Geochemistry Section

Geological Survey of Western Australia

Department of Mines and Petroleum

100 Plain Street

East Perth 6004

Western Australia

T: 61892223345

F: 61892223633

E:Paul.MORRIS@dmp.wa.gov.au

\section{Secretary}

David B. SMITH, USGS

Denver Federal Center, MS-973

Denver, Colorado 80225,

USA

T: $1-303-236-1849$

F: 1-303-236-3200

E: dsmith@usgs.gov

\section{AFRICAN ASSOCIATION OF WOMEN \\ IN GEOSCIENCES (AAWG)}

http://www.aawg.org/AAWG/index.php

\section{President}

Professor Ezzoura ERRAMI

Département de Géologie

Faculté des Sciences

Université Chouaïb Doukkali,

BP. 20, 24000 El Jadida,

Morocco

$\mathrm{T}:+212523342325$

F: +21253342187

E: erramiezzoura@aawg.org

\section{Secretary}

Dr. Juliette TEA-YASSI

PETROCI

Bd Carde, BP V194 Abidjan

Cote d'Ivoire

T: +225 $23536450 / 51$

F: +22523525531

E: jtea@petroci.ci

\section{AMERICAN ASSOCIATION OF}

PETROLEUM GEOLOGISTS

\section{(AAPG)}

http://www.aapg.org

\section{Executive Director}

Mr. David K. CURTISS

AAPG Headquarters

P.O. Box 979

Tulsa, OK 74101-0979

USA

T: +19185602639

F: +19185602626

E: dcurtiss@aapg.org
President

Dr. Edward BEAUMONT

AAPG Headquarters

P.O. Box 979, Tulsa, OK 74101-0979 USA

T: +1 9185602639

$\mathrm{F}:+19185602626$

E: tbeaumont@sm-energy.com

AMERICAN GEOSCIENCES INSTITUTE (AGI)

http://www.agiweb.org

\section{Executive Director}

Dr. Patrick LEAHY

American Geosciences Institute

4220 King Street, Alexandria, VA 22302 USA

$\mathrm{T}:+1$ 703-379-2480

$\mathrm{F}:+1$ 703-379-7563

E: pleahy@agiweb.org

President

Mr. Richard M. POWERS

BCI Engineers \& Scientists Inc.

P.O. Box 5467, Lakeland, FL 33807-5467 USA

T: $01+(863) 667-2345$

F: $01+(863) 667-2662$

E: rpowers@bcieng.com

\section{AMERICAN GEOPHYSICAL UNION}

(AGU)

http://www.agu.org

Executive Director

Ms. Christine MCENTEE

2000 Florida Ave. N.W.

Washington, DC 20009 USA

$\mathrm{T}:+12027777510$

$\mathrm{F}:+12023280566$

E: cmcentee@agu.org

President

Michael MCPHADEN

NOAA/PMEL

7600 Sand Point Way NE

Seattle, WA 98115 USA

$\mathrm{T}:+12065266783$

E: michael.j.mcphaden@noaa.gov

\section{ARAB GEOLOGIST ASSOCIATION} (AGA)

Secretary General

Sarmad Shake MAHMOOD

Baghdad, Iraq

T: +964-7906912974, +964-7702659797

E: irqgeoun@yahoo.com

ASSOCIATION INTERNATIONALE POUR L'ETUDE DES ARGILES (AIPEA)

http://aipea.org/

\section{President}

Professor Christopher BREEN

Head of Polymers

Nanocomposites and Modelling

Materials \& Engineering Research Institute

Sheffield Hallam University

Howard Street

Sheffield S1 1WB, United Kingdom

T: +44 (0)1142253008

F: +44(0)1142253501

E: c.breen@shu.ac.uk 
Secretary General

Dr. Daisy Barbosa ALVES

Petroleo Brasileiro S.A Research Center (CENPES)

Rua Horacio Macedo 950

Cidade Universitaria - Ilha do Fundico

Rio de Janeiro - RJ CEP.: 21941-915

Brazil

T: +55 2138656438

F: +55 2138654562

E:daisy@petrobras.com.br

\section{ASSOCIATION OF EUROPEAN}

GEOLOGICAL SOCIETIES (AEGS)

http://www.aegs.org/aegs.html

\section{President}

Prof. Dr. Corina IONESCU

Head of Mineralogy Department

Babes-Bolyai University of Cluj-Napoca

Str. Kogalniceanu 1

RO-400084 Cluj-Napoca,

Romania

T: +40 264405358

F: +40 264591906

E: corinai@bioge.ubbcluj.ro

\section{Secretary}

Prof. Dr. Marco GIARDINO

Centre for Natural Hazards Research

Department of Earth Sciences

Simon Fraser University

Burnaby, British Columbia V5A 1S6

Canada

$\mathrm{T}:+17787827210$

F: +1 7787824198

E: mgiardin@sfu.ca

\section{ASSOCIATION OF GEOSCIENTISTS}

FOR INTERNATIONAL

DEVELOPMENT (AGID)

http://www.bgs.ac.uk/agid/

\section{Executive Director}

Ms Afia AKHTAR

Geological Society of Bangladesh

153 Pioneer Road, Sengunbagicha

Dhaka-1000, Bangladesh

T: +880 2 9349502, +88028312599

F: +88029339309

E: afia@agni.com

Secretary/Treasurer

Dr. A. J. REEDMAN

15, Malvern Road, Mapperley

Nottingham NG12 5GZ,

United Kingdom

T: +44 (0)115962 1063

F: +44 (0)1159607294

E: antony@areedman.wanadoo.co.uk

BALKAN GEOPHYSICAL SOCIETY (BGS)

http://www.AGSerbia.com

\section{President}

Dr. Snezana KOMATINA-PETROVIC

Dimitrija Avramovica 38

11030 Belgrade, Serbia

T: +381112317005

F: +381637409741

E: unabojan@eunet.rs
CARPATHIAN BALKAN GEOLOGICAL

ASSOCIATION (CBGA)

http://www.cbga2010.org/CBGA.htm

\section{President}

Professor Georgios CHRISTOFIDE

School of Geology

Aristotle University, Faculty of Science

Department of Mineralogy-Petrology

Thessaloniki GR-54124 Greece

T: +302310998541

F: +30 2310998549

E: christof@geo.auth.gr

\section{Secretary General}

Professor Spyros PAVLIDES

Department of Geology

Aristotle University, Faculty of Science

Department of Mineralogy-Petrology

Thessaloniki GR-54124, Greece

T: +302310998541

$\mathrm{F}:+302310998549$

E: pavlides@geo.auth.gr

CIRCUM-PACIFIC COUNCIL (CPC)

http://www.circum-pacificcouncil.org/

\section{President}

DR. H. Gary GREENE

California State University

Professor, Marine Geology

Moss Landing Marine Laboratories

8272 Moss Landing Road

Moss Landing, CA 95039

USA

$\mathrm{T}:+1(831)$ 771-4140 (Lab)

$\mathrm{F}:+1$ (408) 633-7264

E: greene@mlml.calstate.edu

\section{Secretariat}

Mrs. Michele REDNER

CPC Secretariat, USGS

12201 Sunrise Valley Drive,

MS-917, Reston, VA 20192

USA

$\mathrm{T}:+17036486645$

F: +17036484227

E: mredner@usgs.gov

COMMISSION FOR THE GEOLOGICAL MAP OF THE WORLD (CGMW)

http://ccgm.free.fr/index_gb.html

\section{President}

Dr. Phillipe ROSSI

BRGM, BP 6009

Avenue Claude Guillemin

F-45160, Orléans Cedex,

France

$\mathrm{T}:+33(0) 1-4707-2284$

$\mathrm{F}:$ +33(0)1-43-36-9518

E: ccgm@club-internet.fr

Secretary General

Dr. Manuel PUBELLIER

77, Rue Claude-Bernard

F-75005, Paris,

France

$\mathrm{T}:+33(0) 1-4707-2284$

$\mathrm{T}:+33(0) 1-4432-2258$

$\mathrm{F}:$ +33(0)1-43-36-9518

E: ccgm@club-internet.fr
COORDINATING COMMITTEE FOR GEOSCIENCE PROGRAMMES IN EAST AND SOUTHEAST ASIA (CCOP)

http://www.ccop.or.th/

Director

Dr. HE Qingcheng

CCOP Technical Secretariat

CCOP Building

75/10 Rama VI Rd., Phayathai

Ratchathewi, Bangkok 10400, Thailand

$\mathrm{T}:+662-664-5468$

$\mathrm{F}:+(662) 6645429$

E: ccopts@ccop.or.th; hqingcheng@ccop.or.th

DRILLING, OBSERVATION AND

SAMPLING OF THE EARTH'S

CONTINENTAL CRUST (DOSECC)

http://www.dosecc.org

President

Mr. Dennis NIELSON

P.O. Box 58857

675 South Arapeen Drive, Suite 201

Salt Lake City, UT 84158-0857 USA

$\mathrm{T}:+1801583-2150$

$\mathrm{F}:+1801583-2153$

E:dnielson@dosecc.org

Outreach Director

Dr. David M. ZUR

P.O.Box 58857

675 South Arapeen Drive, Suite 201

Salt Lake City, UT 84108 USA

$\mathrm{T}:+1801583-2150$

$\mathrm{F}:+18015859386$

E:dzur@dosecc.org

EUROPEAN ASSOCIATION FOR THE CONSERVATION OF THE GEOLOGICAL HERITAGE (PROGEO)

http://www.sgu.se/hotell/progeo/

President

Dr. William A.P. WIMBLEDON

University of Bristol, Queens Road

Department of Earth Sciences

B88 Bristol, United Kingdom

T: +44292077 2400

F: +44 2920772412

E: newaberdon@tiscali.uk

Executive Secretary

Dr. Lars ERIKSTAD

Norwegian Institute for Nature research

Gaustadalléen 21

NO-0349 Oslo, Norway

E:lars.erikstad@nina.no

EUROPEAN ASSOCIATION OF GEOSCIENTISTS \& ENGINEERS (EAGE)

http://www.eage.org

President

Dr. John UNDERHILL

POB 59

3990 DB Houten

The Netherlands

$\mathrm{T}:+31-88-995-5055$

F: +31-30-634-3524

E:eage@eage.org 
EUROPEAN ASSOCIATION OF SCIENCE EDITORS (EASE)

http://www.ease.org.uk

President

Dr. Joan Marsh

Associate Publishing Director

Wiley-Blackwell

International House

7 High Street, Ealing Broadway

London W5 5DB, UK

E: jmarsh@wiley.com

Secretary

Mrs. Mary Hodgson

P.O. Box 6159

Reading RG19 9DE, United Kingdom

T: +44 (0) 1189700322

F: +44 (0) 1189700322

E: secretary@ease.org.uk

\section{EUROPEAN FEDERATION OF}

GEOLOGISTS (EFG)

http://www.eurogeologists.de

President

Dr. Ruth Allington

C/O Service Géologique de Belgique

Rue Jenner 13, B-1000 Brussels, Belgium

E: efg.president@eurogeologists.eu

\section{Office Director}

Dr. Isabel Fernandez Fuentes

c/o Service Géologique de Belgique

Rue Jenner 13

B-1000 Brussels, Belgium

T: +3227887636

F: +3226477359

E: isabel.fernandez@eurogeologists.eu

\section{EUROPEAN GEOPARKS NETWORK} (EGN)

http://www.europeangeoparks.org

\section{Coordinator}

Íickolas C. ZOUROS

Natural History Museum of the Lesvos

Petrified Forest

GR 81103 Sigri,

Lesvos Island, Greece

$\mathrm{T}:+302253054434$

F: +302253054434

E:nzour@aegean.gr

EUROPEAN GEOLOGICAL SURVEYS

\section{(EGS)}

http://www.eurogeosurveys.org

\section{Secretary General}

Luca Demichel

36-38, Rue Joseph II

1000 Brussels, Belgium

$\mathrm{T}:+3228887553$

F: + 3225035025

E:luca.demicheli@eurogeosurveys.org

\section{EUROPEAN MINERALOGICAL UNION} (EMU)

http://www.univie.ac.at/Mineralogie/EMU

President

Prof. Roberta OBERTI
Consiglio Nazionale della Ricerche

Istituto di Geoscienze e Georisorse

Via Ferrata 1

27100 Pavia, Italy

T: +390382985885

E: oberti@crystal.unipv.it

\section{Secretary}

Prof. Dr. Herta Silvia EFFENBERGER

Institute für Mineralogie und Kristallographie

University of Vienna

Althanstrausse 14

AT-1090 Wien, Austria

T: +43 1 4277/ 53241

F: +4314277/ 9532

E: herta.silvia.effenberger@univie.ac.at

GEOCHEMICAL SOCIETY (GS)

http://www.geochemsoc.org/

\section{President}

Dr. Samuel MUKASA

Dept. of Geological Sciences

2534 CC Little Bldg.

1100 North University Ave

Ann Arbor, MI 48109-1005 USA

T: 734.936 .3227

E: mukasa@umich.edu

E:sam.mukasa@unh.edu

\section{Secretary}

Dr. Neil STURCHIO

Dept of Earth and Environmental Sciences

University of Illinois at Chicago

845 Taylor Street

Chicago Illinois, 606077059 USA

$\mathrm{T}:+13123551182$

F: +13124132279

E: sturchio@uic.edu

\section{GEOLOGICAL SOCIETY OF AFRICA (GSA)}

http://www.geologicalsocietyofafrica.org/

\section{President}

Professor Dr. Aberra MOGESSIE

Institute of Earth Sciences,

Karl-Franzens University of Graz

Universitaetsplatz 2, A-8010 Graz

Austria

$\mathrm{T}:+4310316 / 3805523$

F: +431 0316/380 9865

E: aberra.mogessie@uni-graz.at;

mogessie@uni-graz.at

\section{Secretary General}

Dr. Hassina MOURI

University of Johannesburg

Department of Geology

P.O. Box 524

Gauteng, Auckland Park 2006

South Africa

$\mathrm{T}:+27115593233$

E:hmouri@uj.ac.za

\section{GEOLOGICAL SOCIETY OF AMERICA}

(GSA)

http://www.geosociety.org

\section{Executive Director}

Dr. John W. HESS

The Geological Society of America
3300 Penrose Place

P.O. Box 9140

Boulder, CO 80301 USA

$\mathrm{T}:+13034472020$

$\mathrm{F}:+13033571070$

E: jhess@geosociety.org

International Secretary

Wesley HILL

International Secretariat

Geological Society of America

3300 Penrose Place

P.O. Box 9140

Boulder, Colorado 80301 USA

$\mathrm{T}:+1$ 303.357.1005

$\mathrm{F}:+1$ 303.357.1070

E:whill@geosociety.org

GEOLOGICAL SOCIETY OF INDIA (GSI)

http://www.geosocindia.org

President

Prof. Harsh K. GUPTA

Member, NDMA

Government of India

NDMA Bhawan, A-

Safdarjung Enclave

New Delhi - 110 029, India

$\mathrm{T}:+91-011-26701738$

F: +9-011-26701769

E: harshg123@gmail.com

\section{Secretary}

R.H. SAWKAR

No.63, 12th Cross, Basappa Layout

Gavipuram P.O. Box 1922

Gavipuram, Bangalore - 560 019, India

$\mathrm{T} \cdot+91-080-22422943$

F: +91-080-26613352

E: gsocind@gmail.com

Foreign Secretary

Dr Rajasekhariah SHANKAR

Department of Marine Geology

Mangalore University

Mangalagangotri - 574199 India

T: +91-824-2284465

F: +91-824 2287389

E: rshankar_1@yahoo.com

GEOLOGISCHE VEREINIGUNG (GV)

http://www.g-v.de

\section{Chairman}

Professor/Dr. Ralf LITTKE

Lehrst.f.Geologie,Geochemie u.

Lagerst.d.Erdöls, RWTH Aachen

Lochnerstr. 4-20, 52056 Aachen

Germany

T: $+49 / 241 / 8095748$

F: $+49 / 241 / 8092152$

E:lek@lek.rwth-aachen.de

Secretariat

Mrs. Rita Spitzlei

Geologische Vereinigung

Vulkanstrasse 23

DE-56743 Mendig,

Germany

$\mathrm{T}:+49-2652-989360$

F: +49-2652-989361

E:info@g-v.de; geol.ver@t-online.de 
INTERNATIONAL ASSOCIATION FOR MATHEMATICAL GEOSCIENCES (IAMG)

http://www.iamg.org

\section{President}

Vera Pawlowsky GLAHN

Universitat de Girona

Dpt. D'Informatica i Matematica Aplicada

Campus Montilivi P4

E-17071 Girona, Spain

T: +34972418170

F: +34 972418792

E: vera.pawlowsky@udg.edu

Secretary General

Dr. Daniel M. TETZLAFF

Schlumberger-Information Solutions

5599 San Felipe Ave., Suite 1700

Houston, TX 77056 USA

$\mathrm{T}:+17135132182$

F: +1 7139724855

E: danieltetzlaff@aol.com

INTERNATIONAL ASSOCIATION OF ENGINEERING GEOLOGY AND THE ENVIRONMENT (IAEG)

http://www.iaeg.info/

President

Dr. Carlos DELGADO

E: carlos.delgado@upm.es

Secretary General

Dr. FAQUAN Wu

E:wufaquan@mail.igcas.ac.cn

\section{INTERNATIONAL ASSOCIATION OF} GEOCHEMISTRY (IAGC)

http://www.iagc.ca

President

Dr. Russell S HARMON

Environmental Sciences Division

Army Research Office

(AMSRD-ARL-RO-EV)

U.S. Army Research Laboratory

P.O. Box 12211, Research Triangle Park,

NC 27709-2211, USA

$\mathrm{T}:+1-919-549-4326$

F: +1-919-549-4310

E: russell.harmon@us.army.mil

\section{Secretary}

Dr. Thomas BULLEN

U.S. Geological Survey

Water Resources Division

345 Middlefield Rd. MS 420

Menlo Park, CA 94025 USA

$\mathrm{T}:+16503294577$

F: +1 6503294538

E: tdbullen@usgs.gov

INTERNATIONAL ASSOCIATION OF GEOMORPHOLOGISTS (IAG)

http://www.geomorph.org

President

Dr. Michael CROZIER

Victoria University of Wellington

Institute of Geography

School of Earth Sciences

PO Box 600
Wellington, New Zealand

T: +64 (0)4 4635279

F: +64 (0)4 4635186

E: michael.crozier@vuw.ac.nz

\section{Secretary}

Prof. Morgan DE DAPPER

University of Ghent

Department of Geography

Krijgslaan, 281 (S8)

B-9000-Gent, Belgium

T: +3292644690

F: +3292644982

E: morgan.dedapper@UGent.be

INTERNATIONAL ASSOCIATION

OF HYDROGEOLOGISTS (IAH)

http://www.iah.org

President

Dr. Wilhelm STRUCKMEIER

Federal Institute for Geosciences and Natural

Resources, Stilleweg 2

Postfach 510153, DE-30655 Hannover

Germany

T: +495116432366

F: +49511643 3661

E: wilhelm.struckmeier@bgr.de

\section{Secretary General}

Dr. Shaminder PURI

c/o IAH Secretariat

P.O. Box 4130, Goring

Reading, RG8 6BJ, United Kingdom

T: +448707624462

F: +44870 7628462

E: ShammyPuri@aol.com

Executive Manager

Dr. John CHILTON

IAH Secretariat

PO Box 4130, Goring

Reading, RG8 6BJ, United Kingdom

$\mathrm{T}:+448707624462$

F: +44870 7628462

E: jchilton@iah.org;info@iah.org

INTERNATIONAL ASSOCIATION OF SEDIMENTOLOGISTS (IAS)

http://www.iasnet.org/

\section{President}

Prof. Poppe DE BOER

Utrecht University, P.O. Box 80.021

3508 TA, Utrecht,

The Netherlands

E: pdeboer@geo.uu.nl

\section{Secretary General}

Prof. Vincenzo PASCUCCI

Universita' di Sassari,

Via Piandanna 4

07100 Sassari, Italy

E: pascucci@uniss.it

INTERNATIONAL ASSOCIATION ON THE GENESIS OF ORE DEPOSITS (IAGOD) http://www.iagod.org

President

Prof. Nigel COOK

5005 North Terrace
School of Earth \& Environmental Sciences

The University of Adelaide

Adelaide, SA Australia

T: +61883130686

F: +6188303 4347

E: nigel.cook@adelaide.edu.au

Secretary General

Prof. Sergei CHERKASOV

Vernadsky SGM

Russian Academy of Sciences

11-2 Mokhovaya Street

Moscow, 125009 Russia

$\mathrm{T}:+74952034667$

F: +7 4952035287

E: sergy@sgm.ru

INTERNATIONAL CENTER FOR TRAINING AND EXCHANGES IN THE GEOSCIENCES (CIFEG)

http://www.cifeg.org

President

Prof. Joël ROLET, CIFEG

3, avenue Claude-Guillemin BP 36517, F-45065 Orléans Cedex 2

France

T: +33(0) 238643367

F: +33 (0) 238643472

E: joel.rolet@numericable.fr

Manager

Dr. Guy BENHAMOU, CIFEG

3, Avenue Claude-Guillemin

B.P. 36517

F-45065 Orléans Cedex 2, France

$\mathrm{T}:+33(0) 233643884$

F: +33 (0)238643472

E: g.benhamou@cifeg.org

INTERNATIONAL CONSORTIUM ON LANDSLIDES (ICL)

http://www.iclhq.org/

President

Dr. Paolo CANUTI

Dip. Scienze della Terra

University of Firenze

Via G. La Pira - 4-50121

Firenze, Italy

$\mathrm{T}:+39552757489$

F: +3955218628

E: canuti@geo.unifi.it

Executive Director

Dr. Kyoji SASSA

Research Centre on Landslides

Disaster Prevention Research Institute

Kyoto University, Uji Campus

Uji, Kyoto 611-0011, Japan

$\mathrm{T}:+81774384110$

F: +81774384300

E: sassa@iclhq.org; kyoji.sassa@gmail.com

INTERNATIONAL FEDERATION OF PALYNOLOGICAL SOCIETIES (IFPS)

http://geo.arizona.edu/palynology/ifps.html

President

Dr. Thomas SERVAIS

Laboratoire de Paléontologie et Stratigraphie

Paléontologie et Paléogéographie du Paléozoïque 
UMR 8014 du CNRS

Université des Sciences et Technologies de Lille, Bâtiment SN5

59655 VILLENEUVE D'ASCQ CEDEX

France

T: +33 (0) 320337220

F: +33 (0) 320436900

E:Thomas.Servais@univ-lille1.fr

\section{IUGS Liason}

Dr. Lucy E. EDWARDS

U.S. Geological Survey

926A National Center

Reston, VA 20192, USA

T: +17036485272

F: +17036486383

E: leedward@usgs.gov

\section{Secretary Treasurer}

Dr. Charles H. WELLMAN

Dept. of Animal \& Plant Sciences

University Of Sheffield

Alfred Denny Building, Western Bank

Sheffield S10 2TN, United Kingdom

T: +0114 2223689

F: +0114 2220002

E: c.wellman@sheffield.ac.uk

INTERNATIONAL GEOSCIENCE EDUCATION ORGANISATION (IGEO)

http://www.geoscied.org/

Chair

Dr. Sylke HLAWATSCH

E: kontakt@sylke-hlawatsch.de

\section{Secretary/Treasurer}

Dr. Gabriele OBERMAIER

University of Bayreuth

Bayreuth, Germany

E: Gabriele.Obermaier@uni-bayreuth.de

\section{INTERNATIONAL MEDICAL GEOLOGY} ASSOCIATION (IMGA)

http://www.medicalgeology.org/

\section{Chair}

Prof. Jose CENTENO

Joint Pathology Center

Malcolm Grow Medical Clinic

1057 West Perimeter Road

Bldg 1050; Room GB-33

Joint Base Andrews Naval Air Facility

Washington, MD 20762 USA

$\mathrm{T}:+1$ 240-857-6882

F: +1 240-857-7952

E: jacenteno@comcast.net

Co-Chair for Geosciences

Dr. Olle SELINUS

Swedish Geological Survey

Post Box 670, SE-75128 Uppsala, Sweden

T: +4618 179000

F: +46 185232820

E: olle.selinus@sgu.se

\section{Co-Chair for Medical Science}

Philip WEINSTEIN

School of Population Health

University of Sout Australia

Adelaide, Australia

E:philip.weinstein@unisa.edu.au
INTERNATIONAL MINERALOGICAL

ASSOCIATION (IMA)

http://www.ima-mineralogy.org

\section{President}

Prof. Ekkehart TILLMANNS

Institut fuer Mineralogie und Kristallographie

Geozentrum, Althanstr. 14

A-1090 Wien, Austria

T: +43 (1) 4277-53226

F: +43 (1) 4277-9532

E: kkehart.tillmanns@unvie.ac.at

Past President

Dr. Takamitsu YAMANAKA

Department of Earth and Planetary Science

Osaka University

1-1 Machikaneyama Toyonaka

Osaka 560-0043,

Japan

T: +81668505793

F: +81668505793

E: t.yamanaka@kce.biglobe.ne.jp

\section{Secretary}

Prof. Dr. Richard GOED

Department fur Lithospharenforschung

University Wien, Althanstrasse 14

A-1090 Wien,

Austria

T: +43 (1) 4277-53362

F: +43 (1) 4277-9532

E: richard.goed@unvie.ac.at

INTERNATIONAL PALAEONTOLOGICAL ASSOCIATION (IPA)

http://ipa.geo.ku.edu

President

Prof. Mike BENTON

E:mike.benton@bristol.ac.uk

\section{Secretary General}

Dr. Roger THOMAS

Franklin and Marshall University

Lancaster, PA,

USA

E: roger.thomas@fandm.edu

\section{INTERNATIONAL PERMAFROST}

ASSOCIATION (IPA)

http://www.ipa-permafrost.org/

\section{President}

Dr. Hans-Wolfgang HUBBERTEN

Alfred Wegener Institute

Telegrafenberg A43

D-14773 Potsdam,

Germany

T: +49 3312882100

F: +49 3312882137

E: hans-wolfgang.hubberten@awi.de

\section{Secretariat}

Dr. Hugues LANTUIT

Alfred Wegener Institute

Telegrafenberg A43

D-14773 Potsdam,

Germany

T: +49 3312882162

F: +49 3312882188

E: Hugues.Lantuit@awi.de
INTERNATIONAL SOCIETY FOR ROCK MECHANICS (ISRM)

http://www.isrm.net/

\section{President}

Prof. John A HUDSON

Imperial College London

South Kensington Campus

London SW7 2AZ, United Kingdom

T: +44 1707322819

F: +44 1707375912

E: john.a.hudson@gmail.com

Secretary General

Dr. Luis LAMAS, ISRM Secretariat

c/o Laboratório Nacional de Engenharia Civil Av. do Brasil 101

P-1700-066 Lisboa Codex, Portugal

T: +351 2218443419

F: +3512218443021

E:llamas@lnec.pt; secretariat.isrm@lnec.pt

INTERNATIONAL SOCIETY OF SOIL MECHANICS \& GEOTECHNICAL ENGINEERING (ISSMGE)

http://www.issmge.org/

President

Professor Jean -Louis BRIAUD

Zachry Department of Civil Engineering

Texas A\&M University

College Station, Texas 77843-3136 USA

T:+1- 979-8453795

F:+1 979-8456554

E: briaud@tamu.edu

\section{Secretary General}

Prof. Neil TAYLOR

Geotechnical Engineering Research Centre

City University, Northampton Square

London EC1V 0HB, United Kingdom

T: +44 2074778154

F: +442074778832

E: secretariat@issmge.org

ITALIAN FEDERATION OF EARTH SCIENCES (FIST)

http:www.geoitalia.org

President

Prof. Silvio SENO

Dipartimento di Scienze della Terra e dell'Ambiente

University of Pavia, 27100, Pavia, Italy

T: 39+ 0382-985855

F: 39+ 0382-985890

E: silvio.seno@unipv.it

\section{METEORITICAL SOCIETY}

http://www.meteoriticalsociety.org/

\section{President}

Edward R.D. SCOTT

University of Hawaii at Manoa

Hawaii Institute of Geophysics and Planetology 1680 East-West Road, Post 602

Honolulu, HI 96822 USA

E: president@meteoriticalsociety.org

\section{Secretary}

Gregory HERZOG

Department of Chemistry and Chemical Biology Rutgers University 
610 Taylor Road

Piscataway, New Jersey 08854-8066,

USA

E: secretary@meteoriticalsociety.org

\section{Past President}

Prof. Hiroko NAGAHARA

Dept of Earth and Planetary Science

Graduate School of Science

University of Tokyo

Hongo 7-3-1, Bunkyo-ku

Tokyo 113-0033, Japan

T: +81358414522

F: +81358414569

E: hiroko@eps.s.u-tokyo.ac.jp;

president@meteoriticalsociety.org

\section{NATIONAL GROUND WATER ASSOCIATION (NGWA) \\ http://www.ngwa.org}

\section{Executive Director}

Dr. Kevin B. MCCRAY

601 Dempsey Road

Westerville, Ohio USA

T: +1 6148987791

F: +1 6148987786

E: kmccray@ngwa.org

\section{President}

John W. HENRICH, MGWC

601 Dempsey Road

Westerville, Ohio USA

T: +16148987791

F: +1 6148987786
SOCIETY FOR GEOLOGY APPLIED TO

MINERAL DEPOSITS (SGA)

http://www.e-sga.org/

\section{President}

Dr. F. TORNOS

Instituto Geológico y Minero de España

C/Azafranal 48

37002 Salamanca, Spain

T: +34-923-265009

F: +34-923-265066

E: president@e-sga.org

\section{Executive Secretary}

Dr. Jan PASAVA

Czech Geological Survey

Department of Mineral Resources

Geologická 6

15200 Prague, Czech Republic

T: +420 25817390

F: +42025818748

E: jan.pasava@geology.cz

secretary@e-sga.org

\section{SOCIETY FOR SEDIMENTARY}

GEOLOGY (SEPM)

http://www.sepm.org/

\section{Executive Director}

Dr. Howard HARPER

4111 S Darlington, Suite 100

Tulsa, OK 74135-6373 USA

T: +1 9186103361 (international)

$\mathrm{F}:+19186211685$

E: hharper@sepm.org
President

Chris R. FIELDING

University of Nebraska

Lincoln, NE

USA

E: cfielding2@unl.edu

SOCIETY OF ECONOMIC

GEOLOGISTS, INC. (SEG)

http://www.segweb.org

\section{Executive Director}

Dr. Brian HOAL

7811 Shaffer Parkway

Littleton, CO 80127

USA

T: +1 7209817882 ext. 209

F: +1 7209817874

E: director@segweb.org;

execasst@segweb.org

\section{President}

Dr. M. Stephens ENDERS

E: seg@segweb.org

\section{YOUNG EARTH SCIENCTISTS NETWORK (YES)}

\section{President}

WANG Meng

The University of Leeds

School of Earth and Environment

Leeds, LS2-9JT

United Kingdom

E: networkyes.president@gmail.com

\section{CALL FOR PAPERS}

Episodes is a quarterly science and news journal of the International Union of Geological Sciences (IUGS). It focuses on the publication of results of scientific research and other information addressing issues of interest to the global Earth science community. Special emphasis is given to topics involving geological aspects of population growth and economic development and their resulting impacts on or implications for society. As the principal publication of the IUGS, Episodes also carries information about IUGS scientific programs and activities to the extent necessary to communicate effectively with the worldwide IUGS constituency.

Contributions of the following types of manuscripts are solicited:

- Review papers

- News and views

- Letters to editor
- Conference reports

- Book reviews

- Information on training courses (especially those geared to participants from developing countries)

- Noteworthy new publications, including national or regional geological maps

Episodes also invites photos or other images for the front cover. Photos must be of high technical quality and tell an interesting geological story. A color transparency and one color print (at least $9 \mathrm{~cm} \times 12.6 \mathrm{~cm}$ ) are required for submission, which should be supplemented with a short explanatory paragraph (no more than 100 words).

Please address all contributions to:

Dr. M. Jayananda - Editor

Episodes

Geological Society of India

No.63, $12^{\text {th }}$ Cross, Basappa Layout

Gavipuram P.O., P.O. Box 1922

Bangalore - 560 019, INDIA

Phone: +91-080-22422943; Telefax: +91-80-26613352

Email: episodes.editor@gmail.com 